\title{
(2) Enhanced risk of traumatic brain injury in patients OPEN ACCESS with chronic obstructive pulmonary disease
}

\author{
Tang-Hsiu Huang, ${ }^{1,2}$ Chiung-Zuei Chen, ${ }^{1}$ Hung-I Kuo, ${ }^{1}$ Hong-Ping Er, ${ }^{1}$ \\ Sheng-Hsiang Lin (1) 2,3,4
}

Additional material is published online only. To view please visit the journal online (http://dx.doi.org/10. 1136/jim-2019-001207).

'Division of Chest Medicine, Department of Internal Medicine, National Cheng Kung University Hospital, College of Medicine, National Cheng Kung University, Tainan, Taiwan ${ }^{2}$ Institute of Clinical Medicine, College of Medicine, National Cheng Kung University, Tainan,

Taiwan

${ }^{3}$ Department of Public Health, College of Medicine, National Cheng Kung University, Tainan, Taiwan ${ }^{4}$ Biostatistics Consulting Center, National Cheng Kung University Hospital, College of Medicine, National Cheng Kung University, Tainan, Taiwan

\section{Correspondence to}

Dr Sheng-Hsiang Lin, Tainan, Taiwan;

shlin922@mail.ncku.edu.tw

Accepted 13 December 2019

Published Online First

31 December 2019

\section{ABSTRACT}

This study tests our hypothesis that patients with chronic obstructive pulmonary disease (COPD) have an increased risk of traumatic brain injury (TBI). In this nationwide retrospective cohort study, we used a subset of Taiwan's National Health Insurance Research Database, involving 1 million randomly selected beneficiaries. Patients with newly diagnosed COPD between 2000 and 2008 were identified. They were subgrouped as ' $\mathrm{COPD}_{\mathrm{AE+}}$ ' (if they had severe acute exacerbation of COPD during the follow-ups) or ' $\mathrm{COPD}_{\mathrm{AE-}}$ ' (if they had no acute exacerbation), and were frequency matched with randomly selected subjects without COPD (the 'non-COPD' group). Baseline differences were balanced by the inverse probability of treatment weighting based on the propensity score. For each patient, the risk of TBI during the subsequent 5 years was determined. The competing risk of death was controlled.

We identified 3734 patients in ' $\mathrm{COPD}_{\mathrm{AE}+}$ ', and frequency matched them with 11,202 patients in 'COPD ${ }_{A E-}$ ' and 11,202 subjects in 'non-COPD'. Compared with those in 'non-COPD', patients in 'COPD ${ }_{\mathrm{AE}+}$ ' and ' $\mathrm{COPD}_{\mathrm{AE}-}$ ' had an increased risk of TBI: the adjusted HR for 'COPD ${ }_{A E+}{ }^{\prime}$ was $1.50,95 \% \mathrm{Cl}$ 1.31 to 1.73 , and that for ' $\mathrm{COPD}_{\mathrm{AE}}{ }^{\prime}$ ' was 1.21 , $95 \% \mathrm{Cl} 1.09$ to 1.34. The highest risk was observed in the ' $\mathrm{COPD}_{\mathrm{AE}+}{ }^{\prime}$ group that aged $<65$ (the adjusted HR was $1.92 ; 95 \% \mathrm{Cl} 1.39$ to 2.64$)$.

COPD has been linked to complications beyond the respiratory system. In this study we showed that COPD is associated with an increased risk of TBI.

\section{INTRODUCTION}

Chronic obstructive pulmonary disease (COPD) continues to be a great threat in public health globally. ${ }^{1}$ The debilitating effect of COPD is not confined to the respiratory tract, but can involve multiple organ systems. Cognitive dysfunction, musculoskeletal deconditioning, and impairments in balance and instrumental activities (referring to those activities required to sustain daily living and self-care) of the patients have been well described. ${ }^{1-14}$ These extrapulmonary complications might predispose patients to fall accidents, ${ }^{12-14}$ particularly those who need supplemental oxygen. ${ }^{14}$ Previous studies also demonstrated that COPD might have potentially detrimental effects on the driving performance of the patients, including those without

\section{Significance of this study}

What is already known about this subject?

- Chronic obstructive pulmonary disease (COPD) causes such extrapulmonary complications as sarcopenia and impairments in cognitive, neuromuscular, and balancing functions. These complications predispose the patients to fall and transport-related injury, both of which are epidemiologically leading causes of traumatic brain injury (TBI).

\section{What are the new findings?}

- We showed that patients with COPD of both sex had an increased risk of TBI. The risk was highest in those who had an age of less than 65 years and at least 1 episode of severe acute exacerbation of COPD during the follow-up.

\section{How might these results change the focus} of research or clinical practice?

- Patients and caregivers of COPD need to be aware of such an increased risk of TBI, for TBI would certainly complicate the management and rehabilitation of patients with COPD. Our findings would call for further research to clarify the causal mechanism underlying the association between COPD and TBI, and to develop effective measures to decrease the risk of TBI in this population. 


\section{MATERIALS AND METHODS}

\section{Study objective, design, and population}

In this study, we tested our hypothesis that patients with COPD have an increased risk of TBI. This is a retrospective cohort study using a subset of the National Health Insurance (NHI) Research Database containing comprehensive inpatient and outpatient claims of 1 million beneficiaries randomly selected from Taiwan's 23 million population between 1996 and 2013. All beneficiaries were screened for patients with COPD who were diagnosed between 2000 and 2008. A patient with COPD was defined as one who was 40 years or older, had received the diagnosis of COPD (defined as having such International Classification of Diseases, Ninth Revision, Clinically Modified (ICD-9-CM) codes as 491, 492, and 496) in at least 2 outpatient records (within 1 year apart) or 1 inpatient record, and had received COPD-specific medicinal prescriptions within 2 years that the diagnosis was made. The date when a patient met all these inclusion criteria was defined as the index date for the patient. The codes of all the available prescription medicines used to treat COPD in Taiwan during this same period of time were obtained from the official website of Taiwan's NHI Administration, Ministry of Health and Welfare. ${ }^{38}$ Online supplementary table S1 lists the COPD-specific medicinal prescriptions that were determined according to the contemporary Global Initiative for Chronic Obstructive Lung Disease (GOLD) guidelines parallel in time to our study period. ${ }^{39-41}$ The diagnosis of TBI was specified by such ICD-9-CM codes as 800, 801, 803, 804, and $850-854$, of which 852 and 853 specify hemorrhagic TBI while the others represent non-hemorrhagic TBI. A subject was identified as having an event of TBI when the subject had 1 record of emergency room visit, 1 inpatient record, or 2 outpatient records bearing TBI as a major diagnosis. We excluded patients who had already received the diagnosis of COPD or TBI before the year 2000, those who received the diagnosis of TBI earlier than the diagnosis of COPD, those who were chronically dependent on mechanical ventilation, and those without COPD-specific medicinal prescriptions. We also excluded patients who, within 1 year before and after the diagnosis of COPD, also received the diagnosis of asthma (ICD-9-CM 493) in at least 2 outpatient records or 1 inpatient record. Among all the included patients with COPD, we identified those patients who had at least 1 episode of severe acute exacerbation (defined as an emergency room visit or hospitalization with COPD as the primary diagnosis) ${ }^{121-25}$ after the index date but before the occurrence of TBI or the end of follow-up to constitute the ' $\mathrm{COPD}_{\mathrm{AE}+}$ ' group. Those remaining patients with COPD who had no severe acute exacerbation after the index date during follow-up constituted the 'COPD ${ }_{\mathrm{AE}-}$ ' group. Subjects without COPD were randomly selected from the remaining beneficiaries to constitute the 'non-COPD' group. These 3 groups (namely ' $\mathrm{COPD}_{\mathrm{AE}+}$ ', ' $\mathrm{COPD}_{\mathrm{AE}-}$ ', 'non-COPD') were frequency matched in age, sex, and index date with a ratio of $1: 3: 3$, respectively. For all included subjects, pertinent demographical data were obtained, including age, sex, living area, urbanization level, monthly income, enrollee category (containing 4 categories that served as an approximation of occupational and socioeconomic status: (1) full-time or regularly paid teachers and government employees; (2) employees of privately owned enterprises; (3) other employees or independent professionals; (4) low-income earners, unemployed pensioners, or veterans). Because COPD and TBI were both associated with multiple comorbidities, for each included subject we paid particular attention to identify the presence of coexisting morbidities. Online supplementary table S2 lists the ICD-9-CM codes of all the disease entities included in this study. For all included subjects, data for 5 years following the index date were analyzed to determine the subsequent risk of developing TBI. Death was identified for competing risk analysis. For those who had been hospitalized due to TBI, days of hospital stay were calculated. Subjects missing any of the above-mentioned data, and subjects with insufficient follow-ups, were excluded.

\section{Statistical analysis}

Data were presented as means with SD if normally distributed, and medians with IQR if otherwise. To account for the differences in baseline characteristics and comorbidities among the 3 groups (' $\mathrm{COPD}_{\mathrm{AE}+}$ ', 'COPD $\mathrm{AE}_{\mathrm{AE}}$ ', and 'nonCOPD'), we first compared the baseline characteristics among the 3 groups using Pearson's $\chi^{2}$ test for categorical variables and one-way analysis of variance for continuous variables. We then applied the method of inverse probability of treatment weighting (IPTW) based on the propensity score. ${ }^{42-45}$ Briefly, for each patient the propensity score was calculated by constructing multivariable logistic regression models involving all the demographical characteristics and comorbidities as covariables. The IPTW for each patient was subsequently derived by the inverse of the propensity score, and then transformed to a patient-specific stabilized weight. Stabilized weights were then applied to balance the differences in baseline characteristics and in the prevalence of comorbidities among the 3 study groups, giving rise to an unbiased pseudocohort ('the IPTW cohort'). IPTW was performed separately to balance the differences among the 3 major whole groups and also those among the subgroups. We used the IPTW-balanced data for all subsequent analyses. ${ }^{43}{ }^{45}$ Kaplan-Meier analysis was conducted to determine the 5 -year TBI-free survival probabilities. Cox proportional hazards regression was carried out to derive crude HRs for TBI during follow-up, while stratified Cox regression was performed to derive adjusted HRs, adjusting for such covariates as age, living area, urbanization level, monthly income, enrollee category, and comorbidities. We also controlled for the competing risk of death by constructing competing risk regression models to derive subdistribution HRs. ${ }^{46}$ Sensitivity analysis was done, using R package 'obsSens', by setting an additional hypothetical covariable to evaluate the effect from the potentially unmeasured confounder. All statistical analyses were performed with the statistical packages SAS (V.9.4, SAS Institute) and R (V.3.2.5).

\section{Validation of major diagnoses}

The working definitions we applied in this study to define major diagnoses (COPD and TBI) were validated using the actual medical records at National Cheng Kung University Hospital (a 1200-bed tertiary medical center in southern Taiwan) between January 2008 and December 2011. For COPD, we randomly screened out 200 candidate patients 


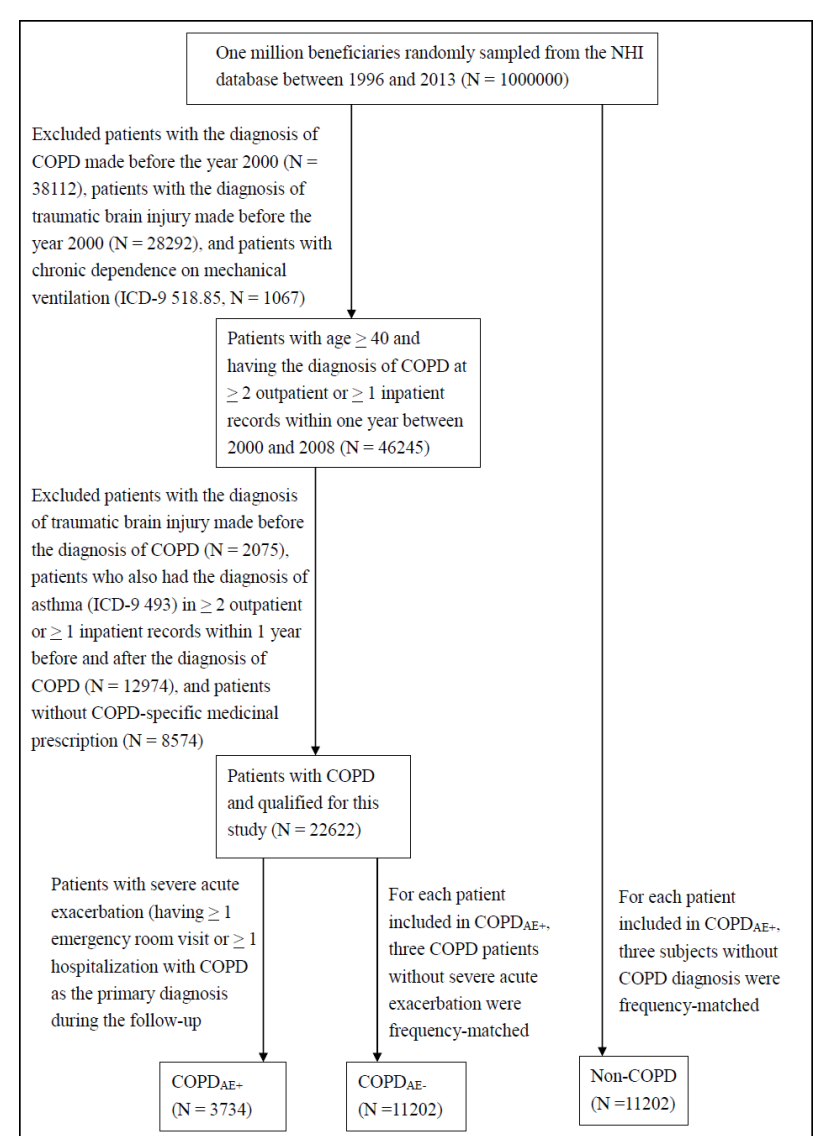

Figure 1 Flow chart of inclusion and exclusion for this study. COPD, chronic obstructive pulmonary disease; ICD-9, International Classification of Diseases, Ninth Revision; NHI, National Health Insurance.

by applying the same working definitions to those we used in this present study (ICD-9-CM codes, age, and the prescription of COPD-specific medication). The diagnoses of these candidate patients were then validated by confirming (through an extensive review of the medical records) the documentation of compatible symptoms, that the diagnosis was made by board-certified pulmonologist, the documentation of GOLD-defined obstructive ventilatory deficit on spirometry, and preferably a positive history of smoking. For TBI, we randomly screened out 200 candidate patients by applying the same working definitions (ICD-9-CM disease codes) as we used in this present study. The diagnoses of these candidate patients were then validated by confirming a documented history of head trauma, the prescription of pertinent managements, and preferably data from brain imaging studies. Positive predictive values (PPV) of the working definitions were 94\% (95\% CI 89\% to 97\%) for COPD and 94\% (95\% CI 90\% to 97\%) for TBI.

\section{RESULTS}

Figure 1 illustrates the flow chart of inclusion and exclusion for this study. From 1 million randomly selected beneficiaries, we identified 3734 patients who had COPD and severe acute exacerbation during follow-up ('COPD $\mathrm{AE}+_{+}$'), and frequency matched them with 11,202 patients with COPD without acute exacerbation ('COPD ${ }_{\mathrm{AE}-}$ ') and 11,202
non-COPD subjects ('non-COPD'). Table 1 displays the baseline characteristics of these 3 groups. Of the included subjects, $65 \%$ were male and more than $70 \%$ aged $\geq 65$ years. Compared with non-COPD subjects, patients with COPD had a higher prevalence of all the selected comorbidities (many of which were also associated with TBI). However, the application of IPTW based on the propensity score effectively balanced those baseline intergroup differences in the prevalence of comorbidities (table 1).

During the 5 -year period after the index date, 312 (8.4\%), 742 (6.6\%), and 588 (5.3\%) patients in 'COPD $\mathrm{AE}+$ ', 'COPD $\mathrm{AE}-$ ', and 'non-COPD' developed TBI, respectively. Patients with COPD, regardless of having severe acute exacerbation or not, consistently exhibited elevated crude and adjusted HRs for TBI relative to nonCOPD subjects: the adjusted HRs for patients with COPD as a whole, and for patients in ' $\mathrm{COPD} \mathrm{AE}_{\mathrm{A}}$ ' and ' $\mathrm{COPD}_{\mathrm{AE}-}$ ' subgroups, were 1.28 (95\% CI 1.16 to 1.41$)$, 1.50 (95\% CI 1.31 to 1.73 ), and 1.21 (95\% CI 1.09 to 1.34 ), respectively. After controlling for the risk of death, multivariable competing risk regression models yielded concordant findings: for patients with COPD as a whole, and for patients in ' $\mathrm{COPD} \mathrm{AE}_{\mathrm{A}}$ ' and ' $\mathrm{COPD} \mathrm{AE}-$ ' subgroups, the subdistribution HRs for TBI were 1.13 (95\% CI 1.02 to 1.23), 1.27 (95\% CI 1.11 to 1.46 ) and 1.08 (95\% CI 0.97 to $1.20)$, respectively. When a comparison was made specifically between the 2 subgroups of patients with COPD, we found that patients in ' $\mathrm{COPD} \mathrm{AE}_{+}$' also had a slightly but significantly higher risk of TBI than those in ' $\mathrm{COPD}_{\mathrm{AE}-}$ ' (the adjusted HR was 1.25 ; 95\% CI 1.09 to 1.43 ), even after controlling for the risk of death (table 2). KaplanMeier analyses revealed the worst TBI-free survival in the group ' $\mathrm{COPD}_{\mathrm{AE}+}$ ', which was followed by ' $\mathrm{COPD}{ }_{\mathrm{AE}-}$ '; non-COPD subjects exhibited the highest probability of TBI-free survival (figure 2).

Further stratifying analysis based on age revealed that patients with COPD (particularly those having severe acute exacerbation), either with advanced $(\geq 65)$ or with younger ( $\geq 40$ and $<65$ ) ages, consistently exhibited increased crude, adjusted, and subdistribution HRs for TBI as compared with non-COPD subjects of the same ranges of age. Moreover, although the study cohort was predominated by patients aged $\geq 65$ years, it was those patients with COPD aged $<65$ years (regardless of having acute exacerbation or not) that exhibited the highest risk of TBI: the adjusted HRs for TBI for patients with COPD as a whole and for patients in ' $\mathrm{COPD}_{\mathrm{AE}+}$ ' and ' $\mathrm{COPD}_{\mathrm{AE}-}$ ' subgroups were 1.73 (95\% CI 1.37 to 2.18 ), 1.92 (95\% CI 1.39 to 2.64$)$, and 1.67 (95\% CI 1.31 to 2.13 ) for patients aged $<65$ years, and 1.23 (95\% CI 1.10 to 1.37$), 1.52$ (95\% CI 1.30 to 1.77 ), and 1.14 (95\% CI 1.01 to 1.28 ) for patients aged $\geq 65$ years, respectively (table 2 ). When the comparison was made between the 2 subgroups of patients with COPD ('COPD ${ }_{\mathrm{AE}+}$ ' vs ' $\mathrm{COPD} \mathrm{AE}-$ '), those patients in ' $\mathrm{COPD}_{\mathrm{AE}+}$ ', regardless of age, exhibited an increased risk of TBI as compared with those in 'COPD $\mathrm{AE}_{-}$' (table 2).

Despite that our cohort had a male predominance, stratifying analysis revealed that both male and female patients with COPD exhibited elevated crude, adjusted, and subdistribution HRs for TBI as compared with non-COPD subjects, particularly those having severe acute exacerbation. When comparing between ' $\mathrm{COPD}_{\mathrm{AE}+}$ ' and ' $\mathrm{COPD}_{\mathrm{AE}-}$ ', 


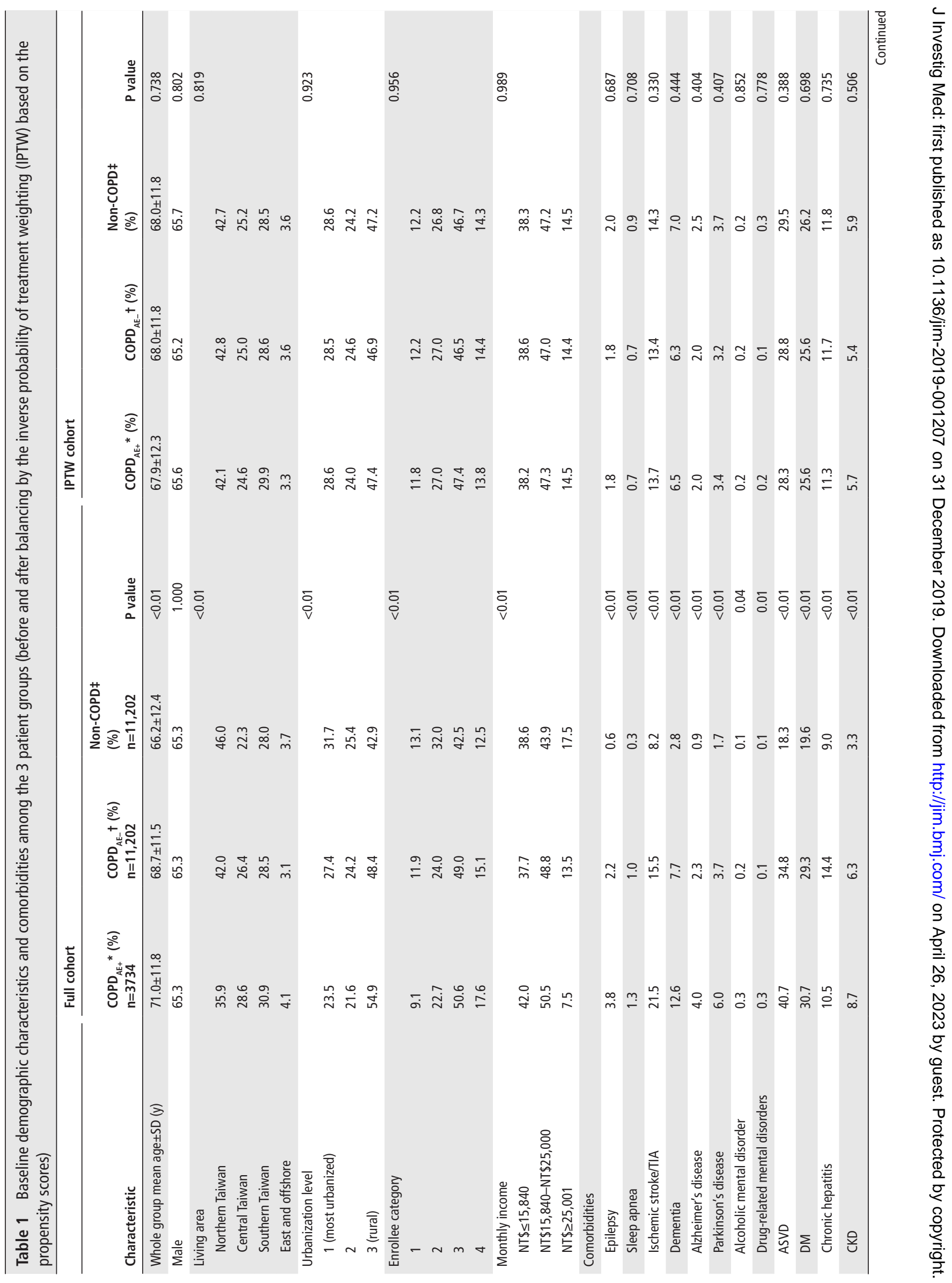




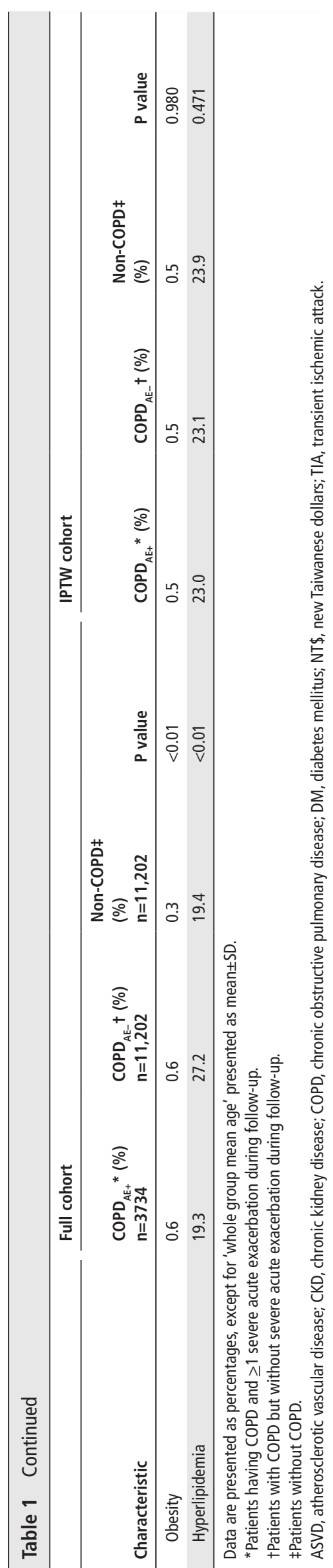

patients of both sexes in ' $\mathrm{COPD}_{\mathrm{AE}+}$ ' had a higher risk of TBI than those in 'COPD ${ }_{\mathrm{AE}-}$ ' (table 3).

We further classified TBI into non-hemorrhagic and hemorrhagic subtypes. Patients with COPD, whether having acute exacerbation or not, had significantly increased risk of non-hemorrhagic TBI as compared with non-COPD subjects, with the adjusted HRs being 1.30 (95\% CI 1.17 to 1.45), 1.61 (95\% CI 1.39 to 1.88 ), and 1.20 (95\% CI 1.07 to 1.35) for all patients with COPD, patients in ' $\mathrm{COPD}_{\mathrm{AE}+}$ ', and patients in ' $\mathrm{COPD}_{\mathrm{AE}-}$ ', respectively. For hemorrhagic TBI, although all the CIs of HRs did not reach statistical significance, the point estimates show several interesting trends. Patients in ' $\mathrm{COPD}_{\mathrm{AE}-}$ ' exhibited a trend of increased risk as compared with non-COPD subjects: the adjusted HR was 1.22 (95\% CI 0.96 to 1.56$)$ for ' $\mathrm{COPD}_{\mathrm{AE}-}$ '. Patients in ' $\mathrm{COPD}_{\mathrm{AE}+}$ ', on the other hand, seemed to exhibit a trend of lower risk of hemorrhagic TBI. When comparing the 2 groups of patients with COPD, we found that patients in ' $\mathrm{COPD}_{\mathrm{AE}+}$ ' still exhibited a significantly increased risk of non-hemorrhagic TBI, but a trend of decreased risk of hemorrhagic TBI, as compared with ' $\mathrm{COPD}_{\mathrm{AE}-}$ '. Multivariable competing risk regression models (controlling for the risk of death) also yielded concordant findings (table 4).

For those patients who developed TBI during follow-up, $111(35.6 \%), 250$ (33.7\%), and 189 (34.4\%) patients in 'COPD $\mathrm{AE}_{+}$', 'COPD $\mathrm{AE}^{\mathrm{A}}$ ', and 'non-COPD' were hospitalized due to TBI, respectively. We found no significant difference in the length of hospital stay among the 3 groups (online supplementary table S3 and figure S1). Sensitivity analysis showed that even when a hypothetical unmeasured confounder was present, COPD (either with or without severe acute exacerbation) was mostly a significant risk factor for TBI at 5 years of follow-up, which further supported the robustness of our findings (online supplementary figure $\mathrm{S} 2 \mathrm{a}, \mathrm{b})$.

\section{DISCUSSION}

COPD is associated with extrapulmonary complications. In this present study, after controlling for the competing risk of death and the differences in baseline characteristics and comorbidities, we show that patients with COPD have an increased risk of TBI, regardless of the differences in sex, age, and the history of acute exacerbation of COPD.

Worldwide epidemiological studies have repeatedly reported fall and transport-related injury as 2 major etiologies of TBI. ${ }^{26-37}$ Specifically, in elderly patients fall accounts for most TBI, whereas in younger adults traffic accidents are the leading cause. ${ }^{30}$ Cognitive dysfunction and weakness of the extremities (particularly lower extremities) have been repeatedly reported as risk factors for fall in elderly patients. ${ }^{47-50}$ The prevalence of COPD increases with advancing age (this was also observed in our study cohort where $>70 \%$ of patients aged $\geq 65$ ). Besides, cognitive dysfunction and cachexia (causing sarcopenia and weakness) are well-recognized complications of COPD. Moreover, previous studies have well demonstrated an impairment in balance among patients with COPD, which predisposed these patients to fall. ${ }^{12-14}$ Therefore, it is likely that an association exists between COPD and fall, and also between COPD and fall-induced TBI. The findings of our present study support for such an association. 


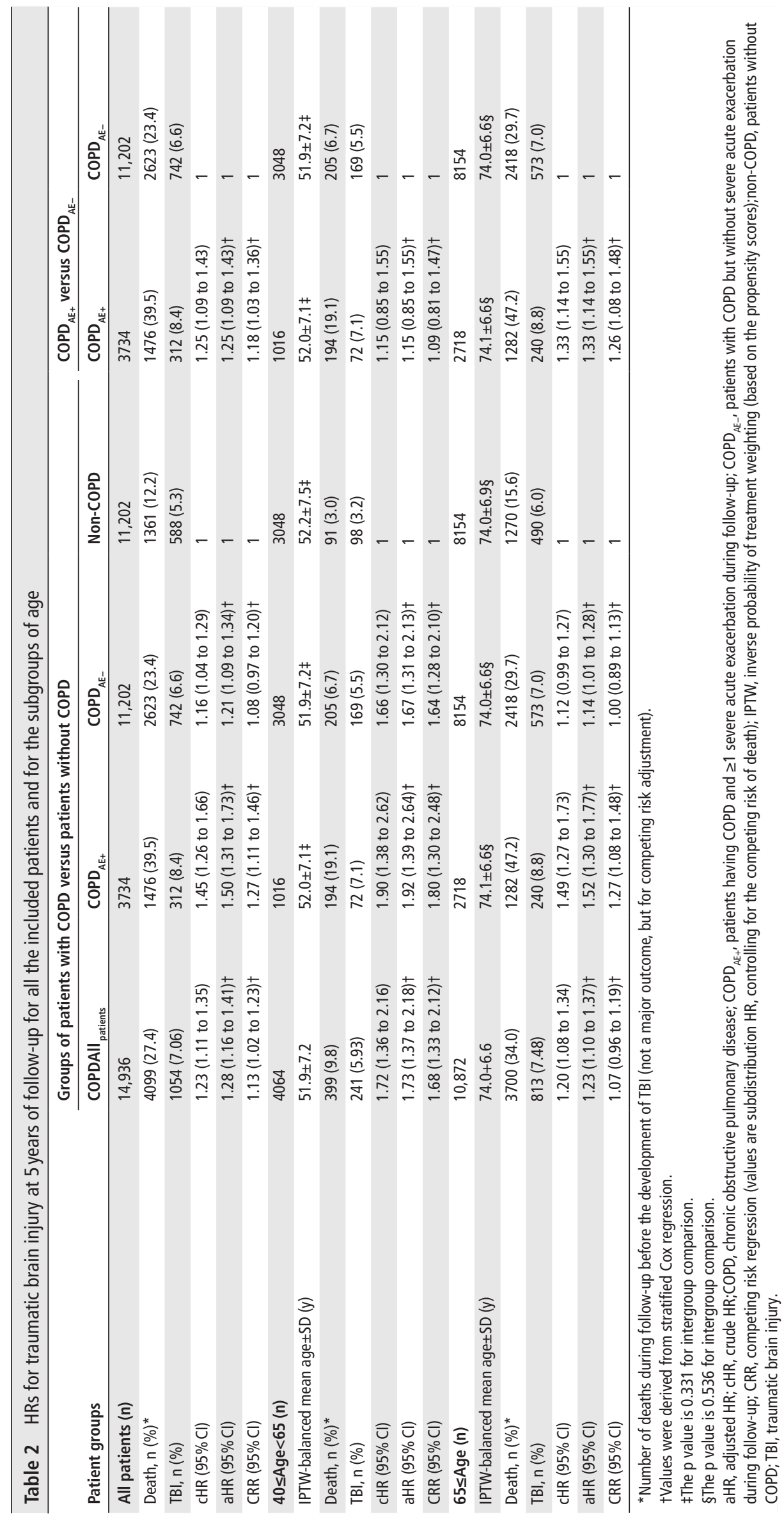




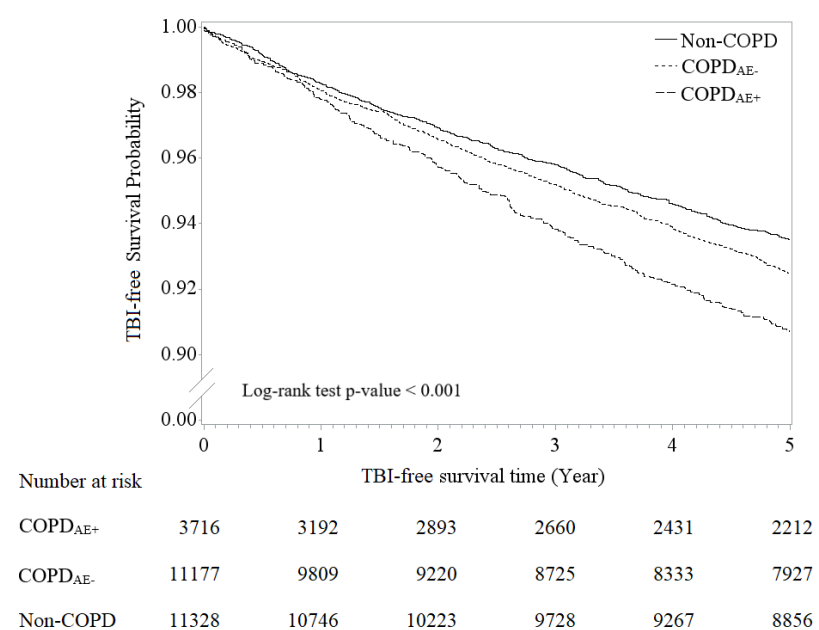

Figure 2 Kaplan-Meier curves of traumatic brain injury (TBI)-free survival rates for the 3 groups of patients (log-rank test $p<0.001$; numbers at risk of the inverse probability of treatment weighting (IPTW) cohort are listed below the plot). COPD, chronic obstructive pulmonary disease; $\mathrm{COPD}_{\mathrm{AE}^{+}}$, patients having COPD and $\geq 1$ severe acute exacerbation during follow-up; $\mathrm{COPD}_{\mathrm{AE}-{ }^{\prime}}$ patients having COPD and no severe acute exacerbation during follow-up; nonCOPD, patients without COPD.

Details about the etiology of individual TBI were unavailable from the NHI Research Database. However, it was possible that in addition to fall, a great proportion of the TBIs of our patients might be transport related. This rationale is based on the international and local epidemiological data. Chiu et $a l^{3536}$ and Lin $e a^{37}$ surveyed the epidemiological characteristics of TBI in Taiwan between 1988 and 2002 and reported that traffic accidents (particularly those relating to riding scooters) accounted for more than $60 \%$ of TBI over this long period of time. As described in the Materials and Methods section, we have validated the working definitions for the major diagnoses for this study using the clinical data of randomly selected 200 patients with COPD and 200 patients with TBI from our hospital. Analysis of the data of these 200 patients for validating the TBI diagnostic definition also identified transport-related injury, in addition to fall, as a major cause of TBI particularly in those aged 40-64 (online supplementary table S4). Previous studies have shown that patients with COPD, even those with mild disease and without hypoxemia, exhibited impairment in their driving performance. ${ }^{15-20}$ Orth et al reported that patients with COPD (with GOLD-defined moderate or severe airflow obstruction but without hypoxemia or hypercapnea) caused more accidents in simulated driving situations than controls. ${ }^{18}$ Interestingly, we observed an even higher risk of TBI in those patients with COPD aged $<65$ years than those aged $\geq 65$ years. Epidemiologically, transport-related injury is more likely than fall to be the cause of TBI among patients of younger age groups. ${ }^{26-37}$ It is possible that both fall and transport-related injury are important causes of TBI in patients with COPD. Nevertheless, further study is warranted to clarify whether there is a direct link between COPD and TBI due to transport-related injury.

Regardless of sex and age strata, patients in ' $\mathrm{COPD}{ }_{\mathrm{AE}+}$ ' consistently exhibited a slightly higher risk of TBI than those in ' $\mathrm{COPD}_{\mathrm{AE}-}$ '. This finding is possibly a reflection of the deleterious impacts of severe acute exacerbations of COPD on the physical and functional performances of the patients. ${ }^{2122}$ However, due to the lack of patient-specific data from the NHI Research Database on changes in ambulatory capacities and functional status following an acute exacerbation of COPD, it is still not possible at this moment, apart from identifying an association, to draw a definite conclusion on the causal relationship between acute exacerbation and the TBI risk. Another interesting finding from our study is that, when compared with patients in ' $\mathrm{COPD}_{\mathrm{AE}-}$ ', those in ' $\mathrm{COPD} \mathrm{AE}_{\mathrm{AE}}$ ' had an increased risk of non-hemorrhagic TBI, but showed a trend toward a reduced risk of hemorrhagic TBI. A possible explanation for this finding is that patients in ' $\mathrm{COPD} \mathrm{AE}_{+}$' might suffer from compromised activity levels (either temporary or prolonged) after the exacerbation event, and might be somehow 'protected' from those physically demanding activities that would potentially cause severe hemorrhagic TBI. Nevertheless, further research is necessary to ascertain whether this explanation is valid.

In our opinion, this present study has several strengths. First, this study investigates a novel topic relating to the

\begin{tabular}{|c|c|c|c|c|c|c|}
\hline \multirow[b]{2}{*}{ Patient groups } & \multicolumn{4}{|c|}{ Groups of patients with COPD versus patients without COPD } & \multicolumn{2}{|l|}{$\mathrm{COPD}_{\mathrm{AE}+}$ versus $\mathrm{COPD}_{\mathrm{AE}-}$} \\
\hline & COPD All patients & $\mathrm{COPD}_{\mathrm{AE}+}$ & $\mathrm{COPD}_{\mathrm{AE}-}$ & Non-COPD & $\mathrm{COPD}_{\mathrm{AE}+}$ & $\mathrm{COPD}_{\mathrm{AE}-}$ \\
\hline All patients (n) & 14,936 & 3734 & 11,202 & 11,202 & 3734 & 11,202 \\
\hline Male (n) & 9748 & 2437 & 7311 & 7311 & 2437 & 7311 \\
\hline $\mathrm{TBI}, \mathrm{n}(\%)$ & $690(7.08)$ & $213(8.7)$ & $477(6.5)$ & $372(5.1)$ & $213(8.7)$ & $477(6.5)$ \\
\hline CHR $(95 \% \mathrm{Cl})$ & $1.25(1.11$ to 1.41$)$ & $1.51(1.27$ to 1.79$)$ & $1.17(1.02$ to 1.33$)$ & 1 & 1.29 (1.09 to 1.53$)$ & 1 \\
\hline $\operatorname{aHR}(95 \% \mathrm{Cl})$ & $1.31(1.16 \text { to } 1.48)^{*}$ & $1.57(1.32 \text { to } 1.87)^{*}$ & $1.23(1.08 \text { to } 1.40)^{*}$ & 1 & $1.28(1.08 \text { to } 1.52)^{*}$ & 1 \\
\hline CRR $(95 \% \mathrm{Cl})$ & $1.14(1.01 \text { to } 1.29)^{*}$ & $1.32(1.11 \text { to } 1.57)^{*}$ & $1.08(0.95 \text { to } 1.24)^{*}$ & 1 & $1.22(1.03 \text { to } 1.45)^{*}$ & 1 \\
\hline Female (n) & 5188 & 1297 & 3891 & 3891 & 1297 & 3891 \\
\hline TBI, n (\%) & $364((7.03)$ & $99(7.6)$ & $265(6.8)$ & $216(5.6)$ & $99(7.6)$ & $265(6.8)$ \\
\hline cHR $(95 \% \mathrm{Cl})$ & 1.20 (1.02 to 1.42$)$ & 1.36 (1.08 to 1.73$)$ & 1.15 (0.97 to 1.37$)$ & 1 & 1.19 (0.94 to 1.50$)$ & 1 \\
\hline aHR $(95 \% \mathrm{Cl})$ & $1.23(1.04 \text { to } 1.44)^{*}$ & $1.39(1.10 \text { to } 1.77)^{*}$ & $1.17(0.98 \text { to } 1.40)^{*}$ & 1 & $1.19(0.94 \text { to } 1.50)^{*}$ & 1 \\
\hline CRR $(95 \% \mathrm{Cl})$ & $1.10(0.93 \text { to } 1.30)^{*}$ & $1.20(0.94 \text { to } 1.52)^{*}$ & $1.07(0.90 \text { to } 1.28)^{*}$ & 1 & $1.12(0.89 \text { to } 1.42)^{*}$ & 1 \\
\hline
\end{tabular}

*Values were derived from stratified Cox regression.

aHR, adjusted HR; CHR, crude HR; COPD, chronic obstructive pulmonary disease; $\mathrm{COPD}_{\mathrm{AE},-}$ patients with COPD but without severe acute exacerbation during follow-up; COPD ${ }_{A E,}$, patients having COPD and $\geq 1$ severe acute exacerbation during follow-up; CRR, competing risk regression (values are subdistribution HR, controlling the competing risk of death);non-COPD, patients without COPD; TBI, traumatic brain injury. 


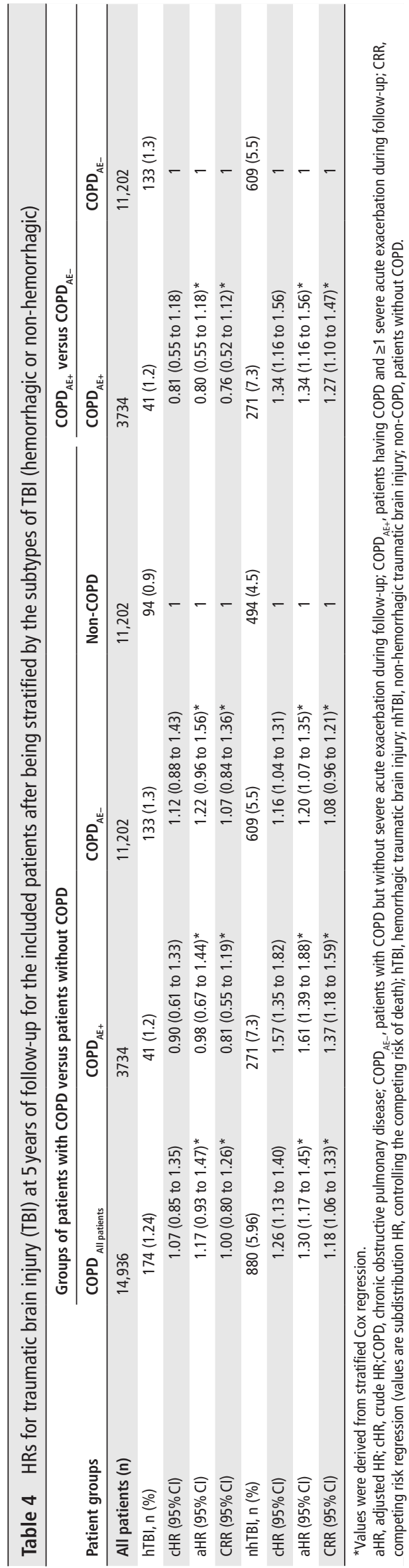

complication of COPD and the care and safety of patients with COPD. Second, findings from this study have important clinical implications. Considering its often long-lasting and devastating consequences, ${ }^{31}$ TBI would certainly complicate the management and rehabilitation of patients with COPD. Patients and caregivers must therefore be aware of such an increased risk of TBI. Moreover, the NHI of Taiwan is a mandatory program, covering $>96 \%$ population in 2000 and consistently $>99 \%$ since $2002 .^{.152}$ The use of the NHI Research Database in this study provided with a random study population of the nationwide scale, which minimized potential selection and referral biases.

Nevertheless, there are limitations to this present study. First, such patient-specific data as spirometric measurements, symptomatic and disease severity, radiographic images, and differential counts of blood cells were unavailable from the NHI Research Database and could not be incorporated to ascertain the diagnosis of COPD. It is possible that those patients with mild COPD who had been managed with only symptomatic treatments (such as antitussive and mucolytic agents) or observation might have been excluded from this study. Besides, despite that we strove to improve the accuracy of the COPD diagnosis by strictly defining our inclusion and exclusion criteria, the overdiagnosis of COPD and the misdiagnosis or miscoding of asthma as COPD in real-world clinical settings still remained possible. However, the accuracy of identifying patients with COPD from the NHI Research Database using working definitions that were similar to those we applied in this study has been validated previously. ${ }^{52}$ Moreover, by performing an independent validation work as was mentioned previously, we demonstrated very high PPVs of our working definitions for COPD as well as for TBI. Therefore, the diagnosis of COPD, and also of TBI, in this present study should be reliable. One issue that must be particularly mentioned is that by strictly excluding those subjects who also received the diagnosis of asthma during the follow-ups, we could enhance the accuracy of the COPD diagnosis, but we might have also excluded patients with asthma-COPD overlap (ACO) who seem to have an increased risk of severe acute exacerbations. ${ }^{5455}$ Further research would help clarify whether patients with $\mathrm{ACO}$ and patients in ' $\mathrm{COPD}_{\mathrm{AE}+}$ ' share a similar risk profile for TBI. Second, the definition we use to identify acute exacerbation of COPD, despite its frequent application in previous studies, ${ }^{24} 2552$ has potential drawbacks. It might have classified those patients with mild or moderate exacerbation (managed by adjusting the medication without emergency visit or hospitalization) into ' $\mathrm{COPD}_{\mathrm{AE}-}$ '. Besides, those patients whose acute exacerbation was secondary to a specific etiology (like pneumonia, ICD-9-CM codes 480-486), or whose exacerbation led to respiratory failure (ICD-9-CM code 518.81), and therefore received a different primary diagnosis than COPD for that hospitalization or emergency room visit, would also be excluded from the study. ${ }^{24}{ }^{25}$ Although our strict definition decreased the number of candidates for ' $\mathrm{COPD}_{\mathrm{AE}+}$ ', we believe it is a necessary step to avoid misclassifying acute events unrelated to COPD into this group. Third, both COPD and TBI are associated with comorbidities. Although in this study we undertook multiple statistical methods to minimize the potentially confounding effects from comorbidities as possible (namely using IPTW based on propensity 
score to balance intergroup differences in comorbidities, adjusting for all the identified comorbidities in multivariate regression analyses, and controlling for the competing risk of death), it remains possible that certain unidentified comorbidities or confounders might have played potential roles accounting for our major findings. However, based on the results of sensitivity analyses (online supplementary figure S2a,b), COPD (either with or without acute exacerbation) remained mostly as a risk factor for TBI even in the presence of a hypothetical unidentified confounder. Therefore, in our opinion, the confounding effect from an unidentified comorbidity (if any) should be small. Fourth, due to the lack of data on the detailed etiologies of TBI and on the serial changes in physical, cognitive, functional, and even behavioral performances of the included patients from the NHI Research Database, it is not possible to establish a definite mechanistic and causal relationship between COPD and TBI. Further investigation is necessary to elucidate the mechanism underlying the association that we found between COPD and the elevated risk of TBI, and also to clarify whether patients with COPD tend to have certain risk-prone behaviors that contribute to the observed increase in the risk of TBI. Fifth, the findings of this study were derived from the clinical data of Taiwan's population, and additional research in other regions studying different ethnic groups would be necessary to confirm whether the increased risk of TBI in patients with COPD is universally observed.

\section{CONCLUSIONS}

In this study, we show that patients with COPD of both sexes have an increased risk of TBI. The risk is higher in those patients with COPD aged $<65$ years than those aged $\geq 65$ years, and also in those patients with COPD who had severe acute exacerbation during follow-up than in those who did not. The findings of our present study add TBI to the list of COPD-associated comorbidities, and raise the need for further research to investigate the underlying mechanism and to determine preventive measures.

Acknowledgements We are grateful to Wan-Ni Chen (MS), statistician from the Biostatistics Consulting Center of National Cheng Kung University Hospital, College of Medicine, National Cheng Kung University, for providing statistical consultation and assistance.

Contributors Conceptualization: THH, CZC, SHL. Methodology: THH, SHL. Software: SHL. Validation: THH, CZC, SHL. Data sorting and analysis: THH, SHL, CZC, HIK, HPE. Writing — original draft preparation: THH. Writing — review and editing: CZC, SHL. Supervision: SHL. Project administration: THH, SHL. Funding acquisition: THH, SHL.

Funding This study was supported by the National Cheng Kung University Hospital, College of Medicine, National Cheng Kung University, under grant NCKUH-10603010.

Disclaimer The funding source did not involve in the study design, the collection, the analysis, and the interpretation of data, as well as the writing of the report, and the decision to submit the article.

Competing interests None declared.

Patient consent for publication Not required.

Ethics approval This study was approved by the Institutional Review Board of National Cheng Kung University Hospital (B-ER-105-326; November 11, 2016).

Provenance and peer review Not commissioned; externally peer reviewed.

Data availability statement Data may be obtained from a third party and are not publicly available. The data which were interpreted in the present study were applied restrictively from the National Health Insurance Research Database (NHIRD), so that this database was used under license limited to the study. In addition, the data are not publicly available. The NHIRD is published by National Health Insurance Administration of Taiwan, in compliance with Taiwan's "Personal Information Protection Act". Requests for data can be submitted as a formal application to the NHIRD (http://nhird.nhri.org.tw).

Author note Parts of the data contained in this paper were presented in a thematic poster in American Thoracic Society 2018 International Conference.

Open access This is an open access article distributed in accordance with the Creative Commons Attribution Non Commercial (CC BY-NC 4.0) license, which permits others to distribute, remix, adapt, build upon this work noncommercially, and license their derivative works on different terms, provided the original work is properly cited, an indication of whether changes were made, and the use is non-commercial. See: http://creativecommons.org/ licenses/by-nc/4.0/.

\section{ORCID iD}

Sheng-Hsiang Lin http://orcid.org/0000-0002-2925-5352

\section{REFERENCES}

1 Vogelmeier CF, Criner GJ, Martinez FJ, et al. Global strategy for the diagnosis, management, and prevention of chronic obstructive lung disease 2017 report. gold executive summary. Am J Respir Crit Care Med 2017;195:557-82.

2 Grant I, Heaton RK, McSweeny AJ. Neuropsychologic findings in hypoxemic chronic obstructive pulmonary disease. Arch Intern Med 1982;142:1470-6.

3 Grant I, Prigatano GP, Heaton RK, et al. Progressive neuropsychologic impairment and hypoxemia. relationship in chronic obstructive pulmonary disease. Arch Gen Psychiatry 1987:44:999-1006.

4 Incalzi RA, Gemma A, Marra C, et al. Chronic obstructive pulmonary disease: an original model of cognitive decline. Am Rev Respir Dis 1993;148:418-24.

5 Liesker JJW, Postma DS, Beukema RJ, et al. Cognitive performance in patients with COPD. Respir Med 2004;98:351-6.

6 Favalli A, Miozzo A, Cossi S, et al. Differences in neuropsychological profile between healthy and COPD older persons. Int I Geriatr Psychiatry 2008;23:220-1.

7 Hung WW, Wisnivesky JP, Siu AL, et al. Cognitive decline among patients with chronic obstructive pulmonary disease. Am I Respir Crit Care Med 2009;180:134-7.

8 Dodd JW, Getov SV, Jones PW. Cognitive function in COPD. Eur Respir J 2010;35:913-22

9 Singh B, Mielke MM, Parsaik AK, et al. A prospective study of chronic obstructive pulmonary disease and the risk for mild cognitive impairment. JAMA Neurol 2014;71:581-8.

10 Roncero C, Campuzano A, Quintano J, et al. Cognitive status among patients with chronic obstructive pulmonary disease. Int I Chron Obstruct Pulmon Dis 2016:11:543-51.

11 Wagner PD. Possible mechanisms underlying the development of cachexia in COPD. Eur Respir J 2008;31:492-501.

12 Butcher SJ, Meshke JM, Sheppard MS. Reductions in functional balance, coordination, and mobility measures among patients with stable chronic obstructive pulmonary disease. J Cardiopulm Rehabil 2004;24:274-80.

13 Chang AT, Seale H, Walsh J, et al. Static balance is affected following an exercise task in chronic obstructive pulmonary disease. I Cardiopulm Rehabil Prev 2008;28:142-5

14 Beauchamp MK, Hill K, Goldstein RS, et al. Impairments in balance discriminate fallers from non-fallers in COPD. Respir Med 2009;103:1885-91.

15 Briggs JE, Patel H, Butterfield K, et al. The effects of chronic obstructive airways disease on theability to drive and to use a roadside alcolmeter. Respir Med 1990:84:43-6.

16 Sung E-J, Min B-C, Kim S-C, et al. Effects of oxygen concentrations on driver fatigue during simulated driving. Appl Ergon 2005;36:25-31.

17 Pretto JJ, McDonald CF. Acute oxygen therapy does not improve cognitive and driving performance in hypoxaemic COPD. Respirology 2008;53:1039-44.

18 Orth M, Diekmann C, Suchan B, et al. Driving performance in patients with chronic obstructive pulmonary disease. J Physiol Pharmacol 2008;59 Suppl 6:539-47.

19 Stressel D, Hegberg A, Dickerson AE, et al. Driving for adults with acquired physical disabilities. Occup Ther Health Care 2014;28:148-53.

20 Skovhus Prior T, Troelsen T, Hilberg O. Driving performance in patients with chronic obstructive lung disease, interstitial lung disease and healthy controls: a crossover intervention study. BMJ Open Resp Res 2015;2:e000092.

21 Pavord ID, Jones PW, Burgel P-R, et al. Exacerbations of COPD. Int J Chron Obstruct Pulmon Dis 2016:11 Spec Iss:21-30. 
22 Ko FW, Chan KP, Hui DS, et al. Acute exacerbation of COPD. Respirology 2016;21:1152-65.

23 Vaz Fragoso CA, Concato J, McAvay G, et al. Respiratory impairment and COPD hospitalisation in older persons: a competing risk analysis. Eur Respir J 2012; 40:37-44

24 Chung W-S, Lin C-L, Kao C-H. Comparison of acute respiratory events between Asthma-COPD overlap syndrome and COPD patients. Medicine 2015:94:e755.

25 Wang M-T, Lo Y-W, Tsai C-L, et al. Statin use and risk of COPD exacerbation requiring hospitalization. Am J Med 2013;126:598-606.

26 Wang CC, Schoenberg BS, Li SC, et al. Brain injury due to head trauma. epidemiology in urban areas of the people's Republic of China. Arch Neurol 1986;43:570-2.

27 Tiret L, Hausherr E, Thicoipe M, et al. The epidemiology of head trauma in Aquitaine (France), 1986: a community-based study of hospital admissions and deaths. Int J Epidemiol 1990;19:133-40.

28 Nell V, Brown DSO. Epidemiology of traumatic brain injury in Johannesburg - II. morbidity, mortality and etiology. Soc Sci Med 1991;33:289-96.

29 Tate RL, McDonald S, Lulham JM. Incidence of hospital-treated traumatic brain injury in an Australian community. Aust N Z J Public Health 1998;22:419-23.

30 Bruns J, Hauser WA. The epidemiology of traumatic brain injury: a review. Epilepsia 2003;44:2-10.

31 Thompson HJ, McCormick WC, Kagan SH. Traumatic brain injury in older adults: epidemiology, outcomes, and future implications. J Am Geriatr Soc 2006;54:1590-5.

32 Harvey LA, Close JCT. Traumatic brain injury in older adults: characteristics, causes and consequences. Injury 2012;43:1821-6.

33 Peeters W, van den Brande R, Polinder S, et al. Epidemiology of traumatic brain injury in Europe. Acta Neurochir 2015;157:1683-96.

34 Whiteman C, Davidov DM, Sikora R, et al. Major trauma and the elder West Virginian: a six year review at a level I trauma center. $W V$ Med $J$ 2016;112:94-9.

35 Chiu WT, Yeh KH, Li YC, et al. Traumatic brain injury registry in Taiwan. Neurol Res 1997;19:261-4.

36 Chiu W-T, Huang S-J, Tsai S-H, et al. The impact of time, legislation, and geography on the epidemiology of traumatic brain injury. J Clin Neurosci 2007;14:930-5.

37 Lin J-W, Tsai S-H, Tsai W-C, et al. Survey of traumatic intracranial hemorrhage in Taiwan. Surg Neurol 2006;66:S20-5.

38 National Health Insurance Administration, Ministry of Health and Welfare of Taiwan, ROC [official homepage on the internet]. Taiwan, ROC.; C 2017, 2017. Available: https://www.nhi.gov.tw/QueryN/Query1.aspx [Accessed 24 Mar 2017].

39 Pauwels RA, Buist AS, Calverley PMA, et al. Global strategy for the diagnosis, management, and prevention of chronic obstructive pulmonary disease. Am J Respir Crit Care Med 2001;163:1256-76.
40 Rabe KF, Hurd S, Anzueto A, et al. Global strategy for the diagnosis, management, and prevention of chronic obstructive pulmonary disease. Am J Respir Crit Care Med 2007:176:532-55.

41 Vestbo J, Hurd SS, Agustí AG, et al. Global strategy for the diagnosis, management, and prevention of chronic obstructive pulmonary disease. $\mathrm{Am} \mathrm{J}$ Respir Crit Care Med 2013;187:347-65.

42 Harder VS, Stuart EA, Anthony JC. Propensity score techniques and the assessment of measured covariate balance to test causal associations in psychological research. Psychol Methods 2010;15:234-49.

43 Xu S, Ross C, Raebel MA, et al. Use of stabilized inverse propensity scores as weights to directly estimate relative risk and its confidence intervals. Value in Health 2010;13:273-7.

44 Leslie S, Thiebaud P. Using propensity scores to adjust for treatment selection bias. Proceedings of the SAS global forum, statistics and data analysis. Cary NC: SAS Institute Inc, 2007. Available: www2.sas.com/proceedings/forum2007/ 184-2007.pdf [Accessed 3 Sep 2018].

45 Austin PC. The use of propensity score methods with survival or time-to-event outcomes: reporting measures of effect similar to those used in randomized experiments. Stat Med 2014;33:1242-58.

46 Lau B, Cole SR, Gange SJ. Competing risk regression models for epidemiologic data. Am J Epidemiol 2009;170:244-56.

47 Nickens H. Intrinsic factors in falling among the elderly. Arch Intern Med 1985; 145:1089-93.

48 Tinetti ME, Speechley M, Ginter SF. Risk factors for falls among elderly persons living in the community. N Eng/ J Med 1988;319:1701-7.

49 Tinetti ME, Inouye SK, Gill TM. Shared risk factors for falls, incontinence, and functional dependence. JAMA 1995;273:1348-53.

50 Muir SW, Gopaul K, Montero Odasso MM. The role of cognitive impairment in fall risk among older adults: a systematic review and meta-analysis. Age Ageing 2012:41:299-308.

51 National Health Insurance Administration, Ministry of Health and Welfare of Taiwan, ROC [official homepage on the internet]. Taiwan, ROC; c.2018. statistical annual reports, 2018. Available: https://www.nhi.gov.tw/English/ Content List.aspx? $n=7 B 24 D 0240347 D A A 8 \&$ topn=BCB2B0D2433F6491 [Accessed 15 Sep 2018].

52 Su VY-F, Yang K-Y, Yang Y-H, et al. Use of ICS/LABA combinations or Lama is associated with a lower risk of acute exacerbation in patients with coexistent COPD and asthma. J Allergy Clin Immunol Pract 2018;6:1927-35

53 VY S, Yang YH, Perng DW, et al. Real-World effectiveness of medications on survival in patients with COPD-heart failure overlap. Aging 2019;11:3650-67.

54 Postma DS, Rabe KF. The Asthma-COPD overlap syndrome. N Engl J Med 2015;373:1241-9.

55 Yanagisawa S, Ichinose M. Definition and diagnosis of asthma-COPD overlap (ACO). Allergol Int 2018;67:172-8. 